\title{
Análisis de confort térmico de la vivienda en valle de San Pedro durante el periodo frío por medio de simulación
}

\author{
Thermal comfort Analysis for the Valley of San Pedro housing during the cold \\ period by use of simulation
}

CAMACHO-IXTA, Ixchel Astrid $\dagger^{*}$, SAHAGUN-VALENZUELA, Miguel I., DELGADO-RENDON, Rene y PÉREZ-LLERENA, Ma. Teresa

Universidad Autónoma de Baja California

ID $1^{\text {er }}$ Autor: Ixchel Astrid, Camacho-Ixta / ORC ID: 0000-0002-2985-6705, Researcher ID Thomson: G-3112-2018, CVU CONACYT ID: 893810

ID $1^{\text {er }}$ Coautor: Miguel I., Sahagun-Valenzuela / ORC ID: 0000-0001-6363-0884, Researcher ID Thomson: G-4120-2018, CVU CONACYT ID: 397669

ID $2^{\text {do }}$ Coautor: Rene, Delgado-Rendon / ORC ID: 0000-0002-5504-3513, Researcher ID Thomson: G-3111-2018, CVU CONACYT ID: 217938

ID $3^{\text {er }}$ Coautor: Ma. Teresa, Pérez-Llerena / ORC ID: 0000-0001-5499-0431, Researcher ID Thomson: Y-8525-2019, CVU CONACYT ID: 483831

DOI: $10.35429 / J A D \cdot 2019.10 .3 .29 .33$

Recibido 25 de Octubre, 2018; Aceptado 18 de Diciembre, 2018

\begin{abstract}
Resumen
Esta investigación se enfoca en los análisis de la problemática del comportamiento de confort térmico interno de una vivienda de interés social, derivados de la falta de adaptaciones al tipo de clima mediterráneo (cálido-seco) predominante de Valle de San Pedro, Tijuana B.C. localizado a latitud $32^{\circ} 29^{\prime} 47^{\prime \prime} \mathrm{N}$ y longitud $116^{\circ} 58^{\prime} 26^{\prime \prime} \mathrm{W}$, condiciones que se presentan en el periodo frío durante diciembre, enero y febrero, cuando se presentan temperaturas mínimas entre $3.5^{\circ} \mathrm{C}$ a $4.4^{\circ} \mathrm{C}$. Se realizó la simulación Design Builder validándola contra datos medidos de la vivienda de manera continua durante los periodos ya mencionados, analizando la temperatura interna de la vivienda. El material de recubrimiento simulado fue el poliestireno, el cual debido a sus características de conductividad térmica permite disminuir el desconfort térmico dentro de la vivienda de interés social contribuyendo a la mejora del espacio habitable, demostrándolo mediante la herramienta de simulación. De esta manera la alternativa de recubrimiento de poliestireno sobre la envolvente de la vivienda, es una solución que permite aumentar las horas de confort dentro del espacio acercando la temperatura interior con respecto a la zona de confort establecida para las condiciones de la localidad durante el periodo frío.
\end{abstract}

Confort térmico, Vivienda de interés social, Design builder, Periodo frío

\begin{abstract}
This research focuses on the analysis of the thermal comfort behavior for the social interest housing, caused by the lack of adaptations to the predominant Mediterranean climate (warm-dry) of San Pedro Valley, Tijuana B.C. located at latitude $32^{\circ} 29^{\prime} 47^{\prime \prime} \mathrm{N}$ and longitude $116^{\circ} 58^{\prime} 26^{\prime \prime} \mathrm{W}$, these conditions occur in the cold period during December, January and February, when minimum temperatures are between $3.5^{\circ} \mathrm{C}$ and $4.4^{\circ} \mathrm{C}$. The Design Builder simulation was carried out by validating results against continuous measured data of the dwelling during the aforementioned periods, analyzing the internal temperature of the dwelling. The simulated coating material was polystyrene, which due to its thermal conductivity characteristics, allows to reduce thermal discomfort in to the social interest housing, contributing to the comfort improvement of the living space, showing it by results of the simulation tool. In this way the polystyrene coating alternative for the housing envelope, is a solution that allows to increase the hours of comfort within the space by bringing the indoor temperature closer to the comfort zone established for the local conditions during the cold period.
\end{abstract}

Citación: CAMACHO-IXTA, Ixchel Astrid., SAHAGUN-VALENZUELA, Miguel I., DELGADO-RENDON, Rene y PÉREZ-LLERENA, Ma. Teresa. Análisis de confort térmico de la vivienda en valle de San Pedro durante el periodo frío por medio de simulación. Revista de Arquitectura y Diseño. 2019, 3-10: 29-33

\footnotetext{
*Correspondencia al Autor (Correo Electrónico: Ixchel_ixta@ hotmail.com)

$\dagger$ Investigador contribuyendo como primer autor.
} 


\section{Introducción}

Hoy en día la problemática del comportamiento térmico dentro de las viviendas de interés social es mucho más común, sumado a la falta de confort térmico derivado por el sistema constructivo y la falta de adaptación al tipo de clima mediterráneo (cálido - seco) de la zona de Valle de San Pedro. Es recurrente que en el periodo frío, de diciembre a enero, se presente este desconfort para los usuarios de la vivienda.

A raíz de este desconfort interior se presentan una serie de problemáticas no nada más de confort, también de salud y económicos, por lo cual el usuario tiene que hacer adaptaciones a la vivienda e inclusive a su vestimenta para poder mitigar este inconveniente.

El objetivo de este trabajo es realizar una simulación con el programa Design Builder para validar los datos medidos de manera física contra los analizados en el programa para constatar que el poliestireno ayuda al confort térmico de la vivienda en el periodo frío.

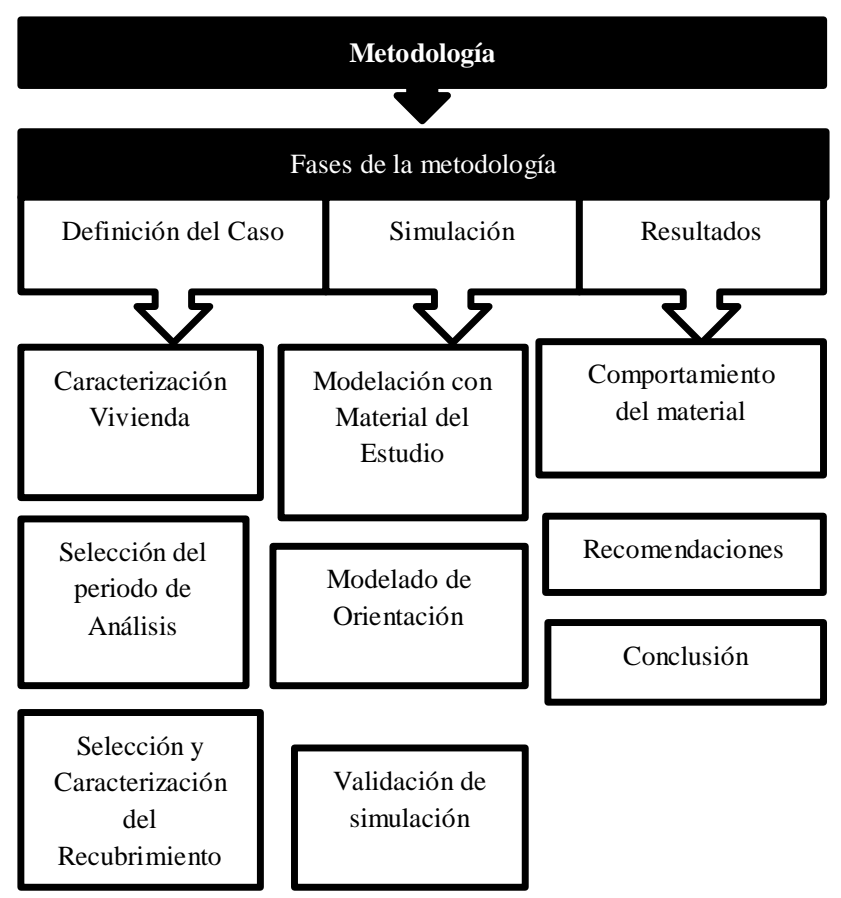

Tabla 1 Metodología

Fuente: Elaboración Propia

\section{Desarrollo}

En el año 2005 se celebró un convenio en conjunto con el XVII ayuntamiento de Tijuana y la empresa constructora de iniciativa privada Urbi.
En el cual, se desarrolló vivienda de interés social donde se realizó el fraccionamiento Valle de San Pedro (Implan, 2005), junto con esto se inicia las problemáticas de desconfort térmico dentro de las viviendas.

Esta problemática que se da en las viviendas de interés social no es exclusiva de estas, también se pueden ver reflejadas en otro tipo de construcciones en Tijuana, deja en claro que los materiales como los sistemas constructivos que se utilizan para sus edificaciones son fundamentales para las condiciones de ambiente que se busca dentro de la vivienda y que es necesario crear al interior un ambiente de confort térmico adecuado a las necesidades climatológicas de la zonas cálidos secos.

\section{Zonificación}

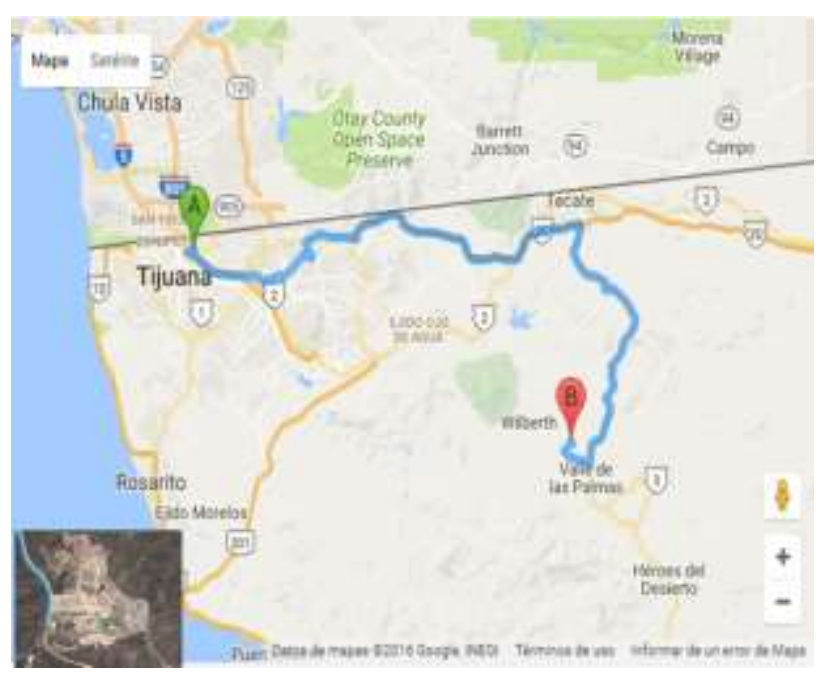

Figura 1 Mapa de Tijuana a Valle de San Pedro Fuente: www.google.map

El estado de Baja California cuenta con cinco municipios cuyos climas son diversos, es decir, van de un clima seco extremo hasta un clima templado húmedo. Tijuana, Baja California debido a su ubicación geográfica se encuentra en las inmediaciones de un clima seco y templado húmedo; podría decirse que en esta ciudad pueden experimentarse en un solo día las cuatro estaciones del año (IMPLAN, 2014) y es en Tijuana donde se localiza Valle de Las Palmas a una latitud $32^{\circ} 29^{\prime} 47^{\prime \prime} \mathrm{N}$ y longitud $116^{\circ} 58^{\prime} 26^{\prime \prime} \mathrm{W}$. 
Una de las características que presentan las viviendas de Valle de San Pedro es la vivienda vertical (Figura 3) con un sistemas constructivos de concreto vaciado (Outinord) el cual presenta propiedades de conductividad térmica muy alto $\left(1.4 \mathrm{~W} / \mathrm{m}^{\circ} \mathrm{C}\right)$ lo cual resulta cambios drásticos en confort térmico dentro de la vivienda.

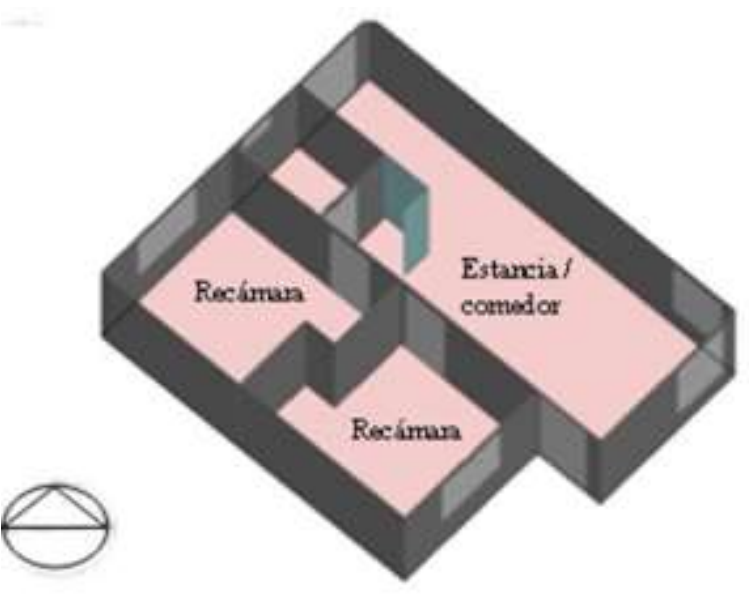

Figura 2 Vivienda de estudio de $63.48 \mathrm{~m}^{2}$ Fuente: Casas URBI

\section{Selección del periodo Análisis}

Para lograr mitigar el desconfort que se presenta por debajo de los $19^{\circ} \mathrm{C}$ y por arriba de $\operatorname{los} 23^{\circ} \mathrm{C}$, que se presenta en el interior de esta vivienda durante el periodo frío, se realizó un análisis del material el cual permite hacer consideraciones para la correcta selección del recubrimiento, de tal manera que ayude a reducir el desconfort al interior de la vivienda y minimicé la utilización de sistemas de calefacción.

El material que se seleccionó para el análisis fue: Poliestireno, el cual se tomó en cuenta por sus propiedades térmicas (tomadas de la base de datos del Ener-Habitat) como conductividad, calor específico, densidad de calor y espesor. Con este material se realizó la simulación y propuesta de recubrimiento, por contar con características adecuadas para el clima de Valle de San Pedro, Tijuana, Baja California.

\section{Selección y Caracterización del Recubrimiento}

El poliestireno cuenta con una conductividad térmica baja comparada a la de otros materiales que pueden usarse para el mismo fin (Tabla 2).
Además de presentar resistencia a la degradación por exposición a la radiación a largo plazo. Uno de sus beneficios es que es higiénico por que no deja que se acumulen microorganismos (no se pudre o se oxida), es ligero y resiste a la humedad, reduce los efectos de emisión de gases de efecto invernadero.

\begin{tabular}{|l|l|l|l|l|}
\hline \multicolumn{5}{|c|}{ Propiedades térmicas y físicas de los materiales } \\
\hline Materiales & $\begin{array}{l}\text { Conductividad } \\
\text { Térmica } \\
\left(\mathrm{W} / \mathrm{m}^{\circ} \mathrm{C}\right)\end{array}$ & $\begin{array}{l}\text { Calor } \\
\text { Especifico } \\
\left(\mathrm{J} / \mathrm{kg}^{\circ} \mathrm{C}\right)\end{array}$ & $\begin{array}{l}\text { Densidad } \\
\left(\mathrm{kg} / \mathrm{m}^{3}\right)\end{array}$ & Espesor \\
\hline $\begin{array}{l}\text { Poliestireno } \\
\text { Concreto } \\
\text { armado }\end{array}$ & 0,035 & 1675 & 50 & 0.025 \\
\hline $\begin{array}{l}\text { Hoja de } \\
\text { yeso }\end{array}$ & 0,1445 & 837 & 2200 & 0.10 \\
\hline
\end{tabular}

Tabla 2 Propiedades Térmicas Fuente: Enerhabitat

\section{Validación de Simulación}

Se ingresaron los datos de conductividad térmica, calor específico, densidad y espesor del recubrimiento en el programa computacional (Design Builder), y las características de la vivienda de estudio a fin de realizar simulaciones de transferencia de calor dependientes del tiempo, y con base a los datos de la estación meteorológica local (archivo epw). De esta manera se toma en cuenta el efecto de la masa térmica y no sólo la resistencia térmica de los materiales o su conductividad. Ello permite diseñar desde avanzadas estrategias de envolvente hasta complejos de sistemas de climatización para enfocarlos a la mejora del confort térmico.

Se evalúa el sistema constructivo mediante el programa de simulación para dos casos: primero con la vivienda sin recubrimiento y segundo implementando el recubrimiento en solo dos lados de la vivienda, en los meses críticos del año (diciembre-enero). Con ello se pudo determinar el comportamiento de la temperatura dentro del edificio.

\section{Resultados}

Para la simulación se consideró que los espacios de la vivienda no se presenta el uso de sistemas de calefacción, y se realizó para un periodo de análisis del 29 de diciembre al 6 de enero (Gráfica 1). 
En la Ilustración 3 se muestra el espacio de estudio modificado con recubrimiento de poliestireno en el sistema constructivo en dos de los muros con contacto directo al exterior. La modificación consistió en añadir una capa exterior de $2.5 \mathrm{~cm}$ de poliestireno al sistema constructivo de concreto de $10 \mathrm{~cm}$.

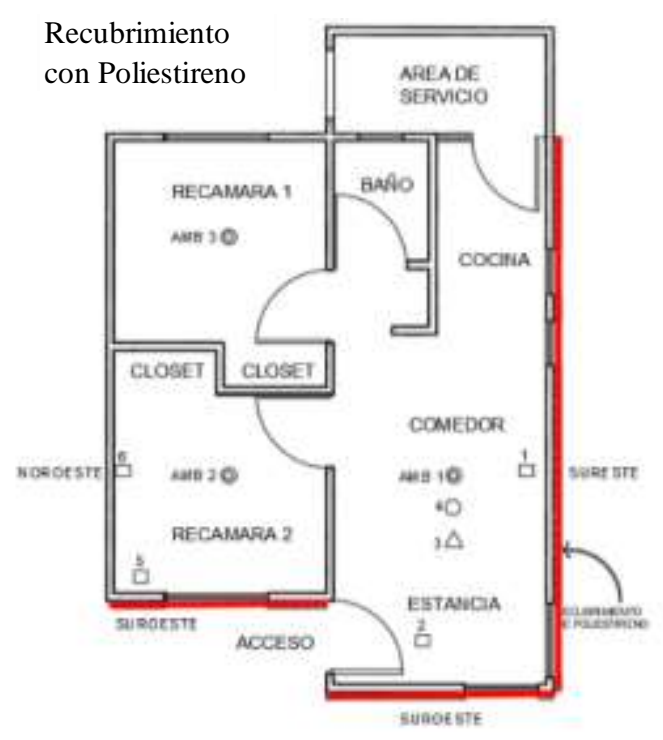

Figura 3 Vivienda de estudio con Poliestireno Fuente: Casas URBI

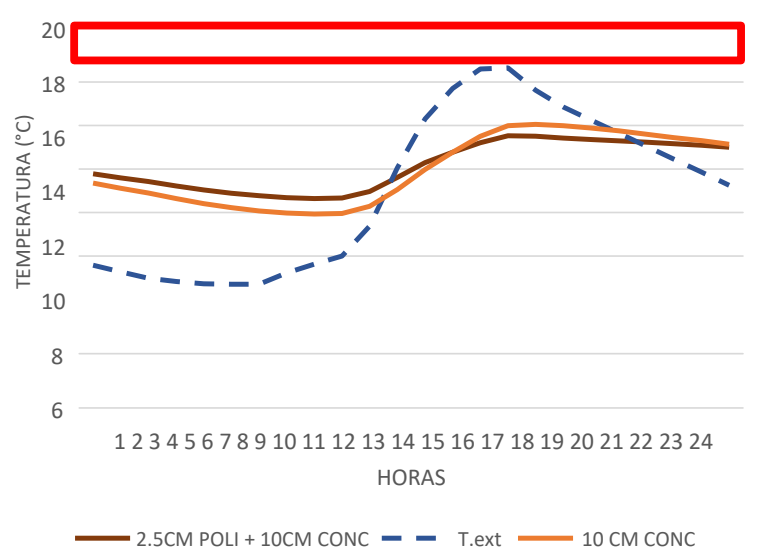

Gráfica 1 Temperatura al interior modificado en un día típico de invierno utilizando una capa de recubrimiento Fuente: Design Builder

En el periodo frío de invierno (diciembre-enero), en la gráfica 1 se aprecia que el muro donde no existe la capa de poliestireno las temperaturas son muy cambiantes ya que por las mañanas temperaturas es muy baja $\left(12^{\circ} \mathrm{C}\right)$ y por las tardes tiende a elevarse $\left(16^{\circ} \mathrm{C}\right)$ manteniendo un rango de temperatura muy amplio.
Sin embargo la que tiene la capa de poliestireno se mantiene un poco más estable $\left(14^{\circ} \mathrm{C}\right.$ y $\left.15^{\circ} \mathrm{C}\right)$ y con un rango menos amplio, lo que permite que al presentarse el aumento de ganancia de calor dentro de la vivienda por actividad humana, equipos y cocina se pueda incrementar la temperatura y aproximarse a la zona de confort sin la necesidad de la implementación de sistemas de calefacción, y con mejor estabilidad térmica dentro de la casa.

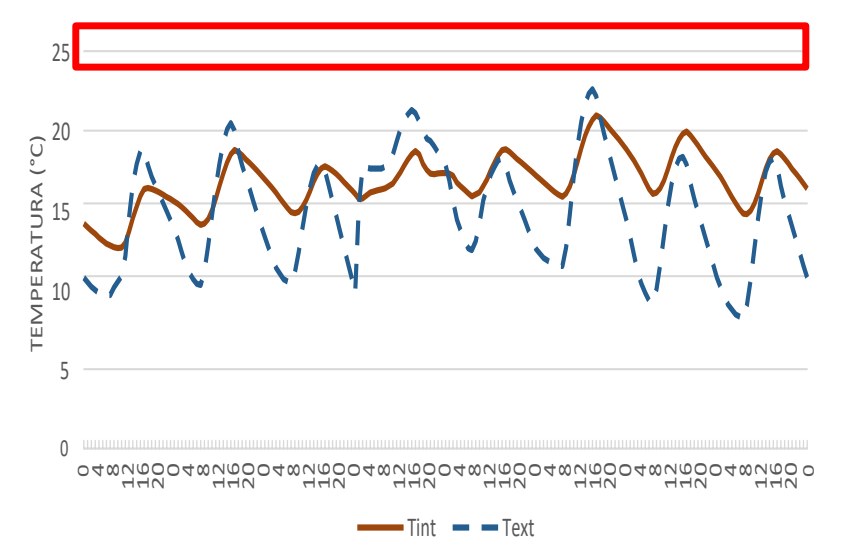

Gráfica 2 Temperatura al interior en una semana típico de invierno sin recubrimiento

Fuente: Design Builder

En la gráfica 2 se presenta la relación entre la temperatura interior y la exterior, en un periodo de 24 horas del periodo de una semana típica (29 diciembre al 6 de enero) de invierno, donde no se contempla algún recubrimiento y se aprecia como la temperatura interior tiene un comportamiento similar al exterior, además se aprecia una respuesta rápida de temperatura interior a los cambios que se dan en la temperatura ambiente del exterior.

\section{Conclusiones}

Se concluye que el material seleccionado (poliestireno) en los muros, contribuye a amortiguar el desconfort que puede presentar una vivienda que está construida de concreto vaciado. En función de los resultados de simulación que presenta el poliestireno, este es el material recomendable para el tipo de vivienda en cuestión y que reduce significativamente el desconfort y proporciona condiciones de estabilidad térmica a la vivienda acercando al usuario al rango de temperatura de confort $\left(19^{\circ} \mathrm{C}-23^{\circ} \mathrm{C}\right)$ debido al comportamiento uniforme de la temperatura dentro de ella. 
Aparte al tener la edificación perfectamente aislada con el poliestireno, se ayuda al ahorro de energía y reducción el uso de climatización no solo en periodo frío ya que el ahorro sería de hasta $40 \%$.

Si se toma en cuenta en el ahorro de climatización para esta época de invierno también hay que tomar en consideración que al tener este recubrimiento. Las familias invertirían menos en cuestiones de salud o inclusive en arropamiento adicional dentro de la vivienda.

El poliestireno, es un material que por su resistencia conviene usar (su espesor es de $2.5 \mathrm{~cm}$ ), ya que permite que la temperatura interior de la vivienda se mantenga de una manera constante durante el periodo crítico de Invierno, con una temperatura entre $14^{\circ} \mathrm{C}$ y $15^{\circ} \mathrm{C}$ el cual al tomar en cuenta la ocupación estos rangos entrarían en la zona de confort de Invierno $\left(19^{\circ} \mathrm{C}\right.$ a $\left.23^{\circ} \mathrm{C}\right)$.

\section{Referencias}

Barrios G, E. P. (2010). Analisis de Indicadores del desempeño térmico de la envolvente de una edificación no climatizada. Memorias de la XXXIV Reunión Nacional de Energía Solar, ANES . Guanajuato, Gto., México.

Barrios G., H. G. (Octubre de 2010). Seccion de Materiales de muros y techos para mejorar el confor térmico en edificaciones no climatizadas. III(3). (P. d. Sonora, Ed.) México, México: Estudios sobre la Arquitectura y Urbanismo del Desierto.

Camacho, I. A. (07 de octubre de 2015). Distribución de la energia solar en un modulo experimental . Campeche, Campeche, México: ANES.

Carrazco, C., \& Morillón, D. (2004). Adecuación Bioclimática de la vivienda de interés social del noroeste de México con base al análisis de la arquitectura vernácula. Avances

Castejón, A. F. (s.f.). Análisis Térmico comparativo por medio de la simulación numérica de tres viviendas de interés social en tres diferentes climas de la república mexicana. México, México. en Energías Renovables y Medio Ambiente, 97-102.
Figueroa, A., Valerdi Madrigal, H., Elías López, P., Tovar Jiménez, E., Castorena Espinosa, G., \& Fuentes Freixanet, V. (2012). Análisis Térmico comparativo por medio de simulación numérica de tres viviendas de interés social en tres diferentes climas de la república mexicana. lema.arq.usin.mx.

Gobierno del Estado de Baja California. (2002). Recuperado el 2016, de Cecytebc: http://cecytebc.edu.mx/spf/compendio_normati vidad/Programas\%20(Nivel\%207)/Programas\% 20Sectoriales/P.\%20Vivienda.pdf

Huelz G., B. G. (2010). Importancia del análisis de transferencia de calor dependiente del tiempo en la evaluación del desempeño térmico de la envolvente de una edificación. (A. 2. Castrejón, Ed.) México, México: Editorial Limusa, UAM Azcapotzalco.

Implantijuana.org. (19 de septiembre de 2014). Recuperado el 08 de junio de 2016, de implantijuana.org:

http://www.implantijuana.org

López, O. A. (2013). El proceso de Transformación de La Vivienda Vernácula en la Región Centro de Guerrero. Guerrero, Guerrero, México.

Pimentel, S. O. (Enero de 2010). Revista RUA. Obtenido de Repositorio Institucional de la Universidad Veracruzana: http://cdigital.uv.mx/bitstream/123456789/3777 1/1/RUA3\%209-13.pdf 